28 Research Square

\title{
Association of Stromal Cell-derived Factor-1 With Diabetic Kidney Disease in Type 2 Diabetic Patients
}

\author{
Chunfeng Lu \\ Nantong City No 1 People's Hospital and Second Affiliated Hospital of Nantong University \\ Jianhua Ma ( $\triangle$ majianhua196503@126.com ) \\ Nanjing Medical University affiliated Nanjing Hospital: Nanjing First Hospital \\ Xueqin Wang \\ Nantong City No 1 People's Hospital and Second Affiliated Hospital of Nantong University \\ Wangshu Liu \\ Nantong City No 1 People's Hospital and Second Affiliated Hospital of Nantong University \\ Xiaoqin Ge \\ Nantong City No 1 People's Hospital and Second Affiliated Hospital of Nantong University
}

Research

Keywords: Type 2 diabetes, stromal cell-derived factor-1, diabetic kidney disease, albuminuria, estimated glomerular filtration rate

Posted Date: January 15th, 2021

DOI: https://doi.org/10.21203/rs.3.rs-144263/v1

License: (c) (1) This work is licensed under a Creative Commons Attribution 4.0 International License. Read Full License 


\section{Abstract}

Background: The present study was designed to explore whether serum stromal cell-derived factor-1 (SDF-1) levels were associated with albuminuria, estimated glomerular filtration rate (eGFR) and diabetic kidney disease (DKD), and detect which clinical parameters might affect serum SDF-1 levels in patients with type 2 diabetes (T2D).

Methods: Serum SDF-1 levels were measured by sandwich ELISA. Patients with an eGFR $<60 \mathrm{ml} / \mathrm{min} / 1.73 \mathrm{~m}^{2}$ and/or a urinary albumin-to-creatinine ratio (UACR) $\geq 30 \mathrm{mg} / \mathrm{g}$ who presented with diabetic retinopathy were identified as having DKD.

Results: Serum SDF-1 levels in T2D patients were significantly higher than those in healthy controls $(p<0.05)$. Urinary albumin and UACR were positively correlated with serum SDF- 1 levels $(r=0.216$ and $=0.276$, respectively, $p<0.01$ ), and eGFR was inversely related with serum SDF- 1 levels $(r$ $=-0.368, p<0.001)$. Moreover, after adjusting for other clinical covariates by multiple linear regression analyses, the serum SDF- 1 levels were independently associated with urinary albumin $(\beta=0.071, t=2.185, p<0.05)$, UACR $(\beta=0.071, \mathrm{t}=2.077, \mathrm{p}<0.05)$ and eGFR $(\beta=-3.975, t=-3.375, p$ $<0.01)$. Furthermore, receiver operating characteristic analysis indicated that the optimal SDF-1 cutoff value for predicting macroalbuminuria was $5.735 \mathrm{ng} / \mathrm{mL}$ (its corresponding sensitivity was $50.00 \%$ and specificity was $81.46 \%$ ), for predicting abnormal albuminuria was $4.321 \mathrm{ng} / \mathrm{mL}$ (its corresponding sensitivity was $58.46 \%$ and specificity was $70.78 \%$ ) and for predicting DKD was $3.505 \mathrm{ng} / \mathrm{mL}$ (its corresponding sensitivity was $83.33 \%$ and specificity was $42.86 \%)$.

Conclusions: The serum SDF-1 levels were positively associated with urinary albumin, UACR and cystatin C, and negatively associated with eGFR, which indicate that SDF-1 may play a critical role in the onset and progression of DKD.

\section{Background}

Diabetic kidney disease (DKD) is one of the most common complications of diabetes and may affect $30 \%$ patients with type 1 diabetes (T1D) and $20 \%$ patients with type 2 diabetes (T2D) [1]. As the leading cause of end stage renal disease (ESRD) worldwide, DKD presumably accounts for about $45 \%$ patients on dialysis [2]. The current management which focused on tight glycemic control and antihypertensive and lipid-lowering failed to prevent the progression of DKD in a large proportion of diabetic patients [3].

Although albuminuria may be the most reliable diagnostic biomarker of DKD, it is not the most perfect one due to various problems [4]. It is possibly widely affected by blood pressure, water intake, infection, fever, recent exercise and so on [5]. Moreover, some patients with normal-albuminuria may suffer advanced renal pathological changes [6]. Therefore, it is of paramount clinical significance to sought new therapeutic targets and markers for DKD.

Stromal cell-derived factor-1 (SDF-1), a member of the CXC chemokine family [7], is ubiquitously expressed in diverse organs and plays multiple function through binding to its receptor CXC chemokine receptor 4 (CXCR4) [8]. SDF-1 is also localized in podocytes and distal tubular cells of human kidney [9], and hyperglycemia can stimulate the secretion of SDF-1 by these cells under diabetic conditions [10]. Elevated SDF-1 can aggregate inflammatory cells to local kidney and regulate the release of inflammatory cytokines, ultimately leading to glomerular sclerosis, loss of podocytes and albuminuria [11]. Hence, SDF-1 is possibly involved in the occurrence and development of DKD. However, few studies revealed the association between serum SDF-1 levels and DKD in Chinese type 2 diabetic patients.

The aim of the present study was to evaluate whether serum SDF-1 levels were related to albuminuria, eGFR and DKD. We also assessed which factors presumably influenced serum SDF-1 levels. Our results demonstrated that serum SDF-1 levels might have the ability to be a therapeutic target and marker for DKD.

\section{Methods}

\section{Study design and participants}

This study was a cross-sectional study, and a total of 171 type 2 diabetic patients were recruited for this study at the Second Affiliated Hospital of Nantong University between May 2020 and November 2020. During the same period, 42 age and gender matched healthy controls from the Department of Physical Examination Center were enrolled. Patients with T2D diagnosed based on the statement of the American Diabetes Association were eligible for inclusion [13]. The exclusion criteria were as follows: (1) T1D; (2) previous drug uses that affect glycemic metabolism, i.e., steroids; (3) previous and current malignant tumors; (4) chronic hepatitis and heart failure; (5) acute diabetic complications, i.e., diabetic ketoacidosis; and (6) other kidney diseases and urinary tract infection which could affect albumin excretion. All subjects agreed to participate in this study, and the study was approved by the medical research ethics committee of Second Affiliated Hospital of Nantong University.

\section{Basic data collection}

Upon enrollment, all subjects completed a questionnaire including parameters on age, sex, weight, height, blood pressure, illness and medical therapy history with the assistance of experienced physicians. Body mass index (BMI) was calculated as the weight/height squared. Blood pressure was measured by a standard mercury sphygmomanometer, and the average of three recordings was recorded. 
Fasting blood samples were collected to measure laboratory parameters. We also collected fresh morning first-void urine samples from type 2 diabetic participants for measurement of urinary albumin and urinary creatinine. UACR was calculated as the ratio of urinary albumin and urinary creatinine. According to UACR, normoabluminuria, microalbuminuria and macroalbuminuria were defined as UACR < 30mg/g, UACR: $30-300 \mathrm{mg} / \mathrm{g}$ and UACR $>300 \mathrm{mg} / \mathrm{g}$, respectively [13]. eGFR was calculated based on the CKD-EPI creatinine-cystatin C equation (2012) [14], and DKD was defined as an eGFR $<60 \mathrm{ml} / \mathrm{min} / 1.73 \mathrm{~m} 2$ and/or a UACR $\geq 30 \mathrm{mg} / \mathrm{g}$ who presented with diabetic retinopathy [15]. All blood samples were centrifuged and stored at $-80^{\circ} \mathrm{C}$. Serum SDF-1 levels were measured by sandwich ELISA (Human SDF-1/CXCL12 Elisa Kit; Elabscience, Wuhan, China). The intra- and interassay coefficients of variation were both less than $10.0 \%$.

\section{Statistical analyses}

Clinical variables are shown for normal controls, type 2 diabetic subjects, and for the quartiles of serum SDF- 1 levels. The mean \pm SD and frequencies (percentages) were adopted to describe normally distributed continuous variables and categorical variables, respectively. Urinary albumin and UACR were log transformed to achieve a normal distribution. We adopted appropriately the one-way analysis of variance (ANOVA) test to compare differences in normally distributed data, the Kruskal-Wallis test to compare differences in skewed distributed data and the chi-square test to compare categorical data among the four subgroups based on the SDF-1 quartiles. The correlations of SDF-1 with Ig (urinary albumin), SDF-1 with Ig (UACR), SDF-1 with eGFR, and SDF-1 with other clinical parameters were analyzed by pearson's or spearman's bivariate correlation analysis as appropriate. Three multiple stepwise linear regression analyses were used to explore to explore the associations of SDF-1 with Ig (urinary albumin), SDF-1 with Ig (UACR), and SDF-1 with eGFR, adjusting for age, gender, diabetic duration, blood pressure, BMl, glycosylated hemoglobin A1c (HbA1c), serum lipid and antidiabetic treatment, as these parameters might affect urinary albumin excretion. Furthermore, receiver operating characteristic (ROC) analysis was conducted to analyze the ability of SDF-1 to indicate macroalbuminuria, abnormal albuminuria and DKD cases, and the cutoff values of SDF-1 to indicate macroalbuminuria, abnormal albuminuria and DKD are provided. Data analyses were performed using SPSS statistical software 18.0 (IBM SPSS Inc., USA). A value of $p<0.05$ was considered to be statistically significant.

\section{Results}

\section{Basic characteristics}

Table 1 displays the clinical characteristics of the participants. Compared with healthy controls, T2D patients had higher SDF-1 concentrations, HbA1c, adenosine deaminase (ADA), triglycerides (TG), white blood cells (WBC) and neutrophil percentage (NEU) (all $p<0.05)$. The mean SDF-1 levels for the whole T2D group were 4.17(0.53, 9.29), and the quartiles were Q1 (<2.95), Q2 (2.95 - 3.92), Q3 (3.94 - 5.40$)$, and Q4 (> 5.40). There were prominent differences in age, urinary albumin, UACR, ADA, blood urea nitrogen (BUN), cystatin C, erythrocyte sedimentation rate (ESR), activated partial thromboplastin time (APTT) and D-dimer among the quartiles of SDF-1 (all $p<0.05$ ). As shown in figure 1 , compared to other quartiles the incidence of microalbumin was higher in the fourth quartile.

\section{Relationships between SDF-1 and clinical parameters in patients with T2D}

As illustrated in the table 2, the serum SDF-1 levels were positively associated with Ig (urinary albumin), Ig (UACR), age, HbA1c, ADA, BUN, cystatin C, DKD incidence, ESR, D-dimer and NEU $(r=0.216, p<0.01 ; r=0.276, p<0.001 ; r=0.256, p<0.01 ; r=0.179, p<0.05 ; r=0.262, p<0.01 ; r=0.353, p<$ $0.001 ; r=0.330, p<0.001 ; r=0.186, p<0.05 ; r=0.262, p<0.01 ; r=0.217, p<0.05 ; r=0.196, p<0.05$, respectively) and negatively associated with eGFR and APTT $(r=-0.368, p<0.001, r=-0.294, p<0.01)$.

\section{Multiple linear regression models displayed independent associations of SDF-1 with albuminuria parameters}

Table 2 shows the associations of SDF-1 with urinary albumin, UACR and eGFR based on multiple linear regression analyses. In the basal unadjusted model 0 , SDF-1 was significantly associated with Ig (urinary albumin) $\left(\beta=0.106, t=3.364, p<0.01\right.$, adjusted $\left.\mathrm{R}^{2}=0.069\right)$, Ig (UACR) $(\beta=0.112, t=$ $3.582, p<0.001$, adjusted $\left.R^{2}=0.076\right)$ and eGFR $\left(\beta=-5.732, t=-4.661, p<0.001\right.$, adjusted $\left.R^{2}=0.129\right)$. After gradually adding the other clinical covariates in each model, we observed a gradual increase in the adjusted $\mathrm{R}^{2}$. In the fully adjusted model 3 , SDF- 1 was still independently associated with Ig (urinary albumin) ( $\beta=0.071, t=2.185, p<0.05$, adjusted $\left.R^{2}=0.207\right)$, Ig (UACR) $\left(\beta=0.071, t=2.077, p<0.05\right.$, adjusted $\left.R^{2}=0.246\right)$ and eGFR $\left(\beta=-3.975, t=-3.375, p<0.01\right.$, adjusted $\left.R^{2}=0.378\right)$. As a result, the serum SDF-1 levels were independently and positively associated with urinary albumin and UACR, and negatively associated with eGFR in type 2 diabetic patients.

\section{ROC analysis to explore the cutoff SDF-1 value to predict macroalbuminuria and abnormal albuminuria}

ROC analysis was further applied to explore the cutoff SDF-1 value to indicate macroalbuminuria, abnormal albuminuria and DKD cases. The optimal cutoff value of SDF-1 to predict macroalbuminuria was $5.735 \mathrm{ng} / \mathrm{mL}$, to predict abnormal albuminuria was $4.321 \mathrm{ng} / \mathrm{mL}$ and to predict DKD was $3.505 \mathrm{ng} / \mathrm{mL}$. The corresponding AUC to predict macroalbuminuria was 0.671 ( $95 \% \mathrm{Cl} 0.626-0.816)$, and its Youden index was 0.315 , its sensitivity was $50.00 \%$, and its specificity was $81.46 \%$ (Fig. 2). The corresponding AUC to predict abnormal albuminuria was 0.639 (95\% $\mathrm{Cl} 0.551-0.726$ ), and its 
Youden index was 0.292 , its sensitivity was $58.46 \%$, and its specificity was $70.78 \%$ (Fig. 3). The corresponding AUC to predict DKD was 0.654 (95\% Cl $0.536-0.773$ ), and its Youden index was 0.262 , its sensitivity was $83.33 \%$, and its specificity was $42.86 \%$ (Fig. 4).

\section{Discussion}

In the present study, we compared the serum SDF-1 levels between the type 2 diabetic patients and healthy controls, analyzed the associations of serum SDF-1 levels with urinary albumin, UACR and eGFR in Chinese type 2 diabetic patients. The main findings of this study are as follows: first, compared with normal controls, serum SDF-1 levels were higher in patients with T2D; second, urinary albumin, UACR and DKD incidence were positively related with serum SDF-1 levels, while eGFR was negatively related with serum SDF-1 levels; third, serum SDF-1 levels were positively associated with HbA1c, D-dimer, ESR, NEU, and negatively associated with APTT; fourth, after adjusting for other clinical covariates, the serum SDF-1 levels were independently and positively associated with urinary albumin and UACR, and inversely associated with eGFR in patients with T2D; and fifth, the optimal SDF-1 cutoff value for predicting macroalbuminuria was $5.735 \mathrm{ng} / \mathrm{mL}$ (its corresponding sensitivity was $50.00 \%$ and specificity was $81.46 \%$ ), for predicting abnormal albuminuria was $4.321 \mathrm{ng} / \mathrm{mL}$ (its corresponding sensitivity was $58.46 \%$ and specificity was $70.78 \%$ ) and for predicting DKD was $3.505 \mathrm{ng} / \mathrm{mL}$ (its corresponding sensitivity was $83.33 \%$ and specificity was $42.86 \%$ ).

The present study demonstrated that serum SDF-1 levels were significantly higher in type 2 diabetic patients than normal controls, and were positively associated with $\mathrm{HbA1c}$. Similar with our study, R. Derakhshan et al revealed that plasm SDF-1 levels were higher in gestational diabetes mellitus mothers than in normal pregnancy mothers [16], and higher in type 2 diabetic patients than in normal controls [17]. SDF-1 and its receptor CXCR4 are expressed in both islet alpha- and beta- cells [18], and the SDF-1/CXCR4 axis may induce islet inflammation by attracting inflammatory cells to the islet and eventually lead to the occurrence of T2D [19]. Hence, SDF-1 may accelerate the onset and progression of T2D.

T2D is capable to result in chronic inflammation characterized by activated mononuclear phagocyte system and increased secretion of cytokines in vivo [20], and inflammation can promote hypercoagulability through the mechanism that cytokines can stimulate the release and expression of procoagulant molecules and inhibit the expression of anti-coagulant molecules [21]. It was shown that serum SDF-1 levels were positively associated with ESR, NEU, D-dimer and negatively associated with APTT in this study. ESR and NEU are recognized inflammatory biomarkers, while APTT and Ddimer are important indicators of coagulation function. A shorter APTT reflects enhanced endogenous coagulation function [22] and D-dimer is a fibrin degradation product [1]. In patients with disseminated intravascular coagulation (DIC), the coagulation activation was able to contribute to increased levels of the circulating SDF-1, which in turn enhanced platelet aggregation and then promoted coagulation [23]. Given these results, elevated serum levels of SDF-1 presumably reflected the condition of hyperglycemia, inflammation and hypercoagulability in type 2 diabetic patients. As DKD is a result of the interaction of hyperglycemia, hemodynamic alterations, inflammation and oxidative stress [24], SDF-1 may play a critical role in the development and progression of DKD, or at least can serve as a potential predictor of DKD. In support of this conclusion, renal biopsy revealed that SDF-1 significantly increased in kidneys of diabetic rodents and patients with DKD [25], and blockade of SDF-1 by the specific inhibitor NOX-A12 significantly reduced podocyte loss and glomerulosclerosis, thereby reducing proteinuria [26].

In the early stage of DKD, renal alterations are manifested as glomerular hypertrophy, glomerular basement membrane (GBM) thickening, podocyte loss and tubular damage. In advanced DKD, renal morphological changes include glomerulosclerosis and tubulointerstitial fibrosis, and corresponding clinical features include renal filtration function declines with or without albuminuria [27]. Our study showed that serum SDF-1 levels were positively associated with urinary albumin and UACR, and negatively associated with eGFR. UACR, an evaluation index of increased urinary albumin, can reflect damage to the basement membrane and endothelium of glomerular capillaries, and represent early changes of DKD [28]. Based on the CKD-EPI creatinine-cystatin C equation, eGFR can accurately estimate renal function [14]. Thus, serum SDF-1 levels may have the potential to be an evaluation indicator of early and advanced DKD. Recent researches strongly suggest that renal tubular damage is a vital component of early DKD and may precede the occurrence of glomerular injury [29]. Serum cystatin C, a non-glycosylated, low molecular weight and basic protein, is completely removed by glomerular filtration and subsequently reabsorbed and degraded by proximal tubular [30]. In addition to the ability to assess GFR, cystatin C can also reflect renal tubular damage of DKD [31]. In this study, serum SDF-1 levels were significantly associated with cystatin C ( $r=$ $0.330, p<0.001)$. Therefore, serum SDF-1 levels possibly and roundly reflect renal alterations of DKD patients, which is consistent with that SDF- 1 is expressed in podocytes and distal tubular cells of human kidney [9].

On the contrary, there are also studies showed that elevated SDF-1 levels may play a role in renal protection via other mechanisms. SDF-1 is critical for the mobilization and migration of endothelial progenitor cells (EPCs), while EPCs can promote endothelial repair and angiogenesis [32]. Therefore, increased SDF-1 may exert a renal protected function by promoting the mobilization and migration of EPCs. SDF- 1 is mainly cleaved and inactivated by dipeptidyl peptidase-4 (DPP-4) enzyme in vivo [33]. DPP-4 inhibitors are a class of commonly used oral antidiabetic drugs [34], and clinical studies have shown that short-term administration of DPP-4 inhibitors can significantly increase plasma SDF- 1 and EPCs levels in type 2 diabetic patients [35-37]. However, EPCs isolated from diabetic patients are structurally and functionally defective [38]. So even though EPCs level is raised, it fails to promote tissue repair and angiogenesis. Milton Packer summarized several large-scale trials of DPP-4 inhibitors, concluding that DPP-4 inhibitors could improve vascular complications of T2D by upregulating GLP-1 levels, but potentiation of SDF-1 undermined the protective effect [39]. All in all, SDF-1 may contribute to the onset and progression of DKD in patients with T2D.

Several limitations of our study should be addressed. First, the present study could not explain the causal relationship between SDF-1 and albuminuria due to the common problem of cross-sectional studies. Second, on account of the small sample size of this study, the correlation

Page $4 / 14$ 
between DPP-4 inhibitors use and plasma SDF-1 levels could not be verified. Third, all the subjects enrolled in this study were Chinese, which limited the wide applicability of our study. Therefore, further research should be conducted to validate the results of our study and to address the above limitations.

\section{Conclusions}

The serum SDF-1 levels were positively associated with urinary albumin, UACR and cystatin C, and negatively associated with eGFR, which indicate that SDF-1 may play a critical role in the onset and progression of DKD.

\section{Abbreviations}

SDF-1: stromal cell-derived factor-1, eGFR: estimated glomerular filtration rate (eGFR), DKD: diabetic kidney disease, T2D: type 2 diabetes, UACR: urinary albumin/creatinine ratio, T1D: type 1 diabetes, ERSD: end stage renal disease, CXCR4: CXC chemokine receptor 4, BMI: body mass index, ANOVA: analysis of variance, HbA1c: glycosylated hemoglobin A1c, ROC: receiver operating characteristic, ADA: adenosine deaminase, TG:

triglycerides, WBC: white blood cell, NEU: neutrophil percentage, BUN: blood urea nitrogen, ESR: erythrocyte sedimentation rate, APTT: activated partial thromboplastin time, DIC: disseminated intravascular coagulation, GBM: glomerular basement membrane, EPCs: endothelial progenitor cells, DPP-4: dipeptidyl peptidase-4, GLP-1: glucagon-like peptide 1

\section{Declarations}

\section{Authors' contributions}

CL participated in the design of the study, data collection, analysis of the data, and drafting of the manuscript. JM and XW conceived of the study, participated in its design and revised the manuscript. WL and XG participated in data collection. All authors read and approved the final manuscript.

\section{Author details}

1 Department of Endocrinology, Nanjing First Hospital, Nanjing Medical University, Nanjing 210012, China. 2 Department of Endocrinology, Affiliated Hospital 2 of Nantong University and First People's Hospital of Nantong City, Nantong 226001, China.

\section{Acknowledgements}

Not applicable.

\section{Competing interests}

The authors declare that they have no competing interests.

\section{Availability of data and materials}

The current data are available to all interested researchers upon reasonable request. Requests for access to data should be made to the principal investigators of the study.

\section{Consent for publication}

Not applicable.

\section{Ethics approval and consent to participate}

The study was approved by the institutional review board of Affiliated Hospital 2 of Nantong University and First People's Hospital of Nantong City, and written informed consent was obtained from all participants.

\section{Funding}

The study was supported by the Medical Research Project of Health Commission of Nantong (MB2020012).

\section{References}

1. Domingueti CP, Dusse LM, Carvalho Md, de Sousa LP, Gomes KB, Fernandes AP. Diabetes mellitus: The linkage between oxidative stress, inflammation, hypercoagulability and vascular complications. J Diabetes Complications 2016;30(4): 738-745

2. Hosny SS, Bekhet MM, Hebah HA, Mohamed NR. Urinary neutrophil gelatinase-associated lipocalin in type 2 diabetes: Relation to nephropathy and retinopathy. Diabetes Metab Syndr 2018;12(6): 1019-1024 
3. Rayego-Mateos S, Morgado-Pascual JL, Opazo-Ríos L, Guerrero-Hue M, García-Caballero C, et al. Pathogenic pathways and therapeutic approaches targeting inflammation in diabetic nephropathy. Int J Mol Sci 2020;21(11): 3798

4. Moresco RN, Sangoi MB, De Carvalho JA, Tatsch E, Bochi GV. Diabetic nephropathy: traditional to proteomic markers. Clin Chim Acta 2013;421: $17-30$

5. American Diabetes Association. Standards of medical care in diabetes. Diabetes Care 2013;36: S11-66

6. Kishore L, Kaur N, Singh R. Distinct Biomarkers for early diagnosis of diabetic nephropathy. Curr Diabetes Rev 2017;13(6): 598-605

7. Zhu Y, Hoshi R, Chen S, Yi J, Duan C, et al. Sustained release of stromal cell derived factor-1 from an antioxidant thermoresponsive hydrogel enhances dermal wound healing in diabetes. J Control Release 2016;238: 114-122

8. Deng L, Jia J, Yao J, Xu Z. Stromal cell-derived factor 1 (SDF-1) and its receptor CXCR4 improves diabetic retinopathy. Biosci Biotechnol Biochem 2019;83(6): 1072-1076

9. Karimabad MN, Hassanshahi G. Significance of CXCL12 in type 2 diabetes mellitus and its associated complications. Inflammation 2015;38(2): 710-717

10. Elmarakby AA, Sullivan JC. Relationship between oxidative stress and inflammatory cytokines in diabetic nephropathy. Cardiovasc Ther 2012;30(1): 49-59

11. Shi H, Lu R, Wang S, Chen H, Wang F, et al. Effects of SDF-1/CXCR4 on acute lung injury induced by cardiopulmonary bypass. Inflammation 2017;40(3): 937-945

12. American Diabetes Association. Diagnosis and classification of diabetes mellitus. Diabetes Care 2011;34: S62-69

13. Sueud T, Hadi NR, Abdulameer R, Jamil DA, Al-Aubaidy HA. Assessing urinary levels of IL-18, NGAL and albumin creatinine ratio in patients with diabetic nephropathy. Diabetes Metab Syndr 2019;13(1): 564-568

14. Inker LA, Schmid $\mathrm{CH}$, Tighiouart $\mathrm{H}$, Eckfeldt JH, Feldman $\mathrm{HI}$, et al. Estimating glomerular filtration rate from serum creatinine and cystatin $\mathrm{C}$. $\mathrm{N}$ Engl J Med 2012;367(1): 20-29

15. Church E, Poppe K, Harwood M, Mehta S, Grey C, et al. Relationship between estimated glomerular filtration rate and incident cardiovascular disease in an ethnically diverse primary care cohort. N Z Med J 2019;132(1491): 11-26

16. Darakhshan S, Fatehi A, Hassanshahi G, Mahmoodi S, Hashemi MS, et al. Serum concentration of angiogenic (CXCL1, CXCL12) and angiostasis (CXCL9, CXCL10) CXC chemokines are differentially altered in normal and gestational diabetes mellitus associated pregnancies. J Diabetes Metab Disord 2019;18(2): 371-378

17. Karimabad MN, Hassanshahi G. Significance of CXCL12 in type 2 diabetes mellitus and its associated complications. Inflammation 2015;38(2): 710-717

18. Aydin Ozgur B, Coskunpinar E, Bilgic Gazioglu S, Yilmaz A, Musteri Oltulu Y, et al. Effects of complement Regulators and chemokine receptors in type 2 diabetes. Immunol Invest 2020;10.1080

19. Derakhshan R, Arababadi MK, Ahmadi Z, Karimabad MN, Salehabadi VA, et al. Increased circulating levels of SDF-1 (CXCL12) in type 2 diabetic patients are correlated to disease state but are unrelated to polymorphism of the SDF-1 $\beta$ gene in the Iranian population. Inflammation 2012 Jun;35(3): 900-904

20. Alicic RZ, Johnson EJ, Tuttle KR. Inflammatory Mechanisms as New Biomarkers and Therapeutic Targets for Diabetic Kidney Disease. Adv Chronic Kidney Dis 2018;25(2): 181-191

21. Margetic, S. Inflammation and haemostasis. Biochemical Medicine 2012;22: 49-62

22. Sun J, Liu C. Correlation of vascular endothelial function and coagulation factors with renal function and inflammatory factors in patients with diabetic nephropathy. Exp Ther Med 2018;16(5): 4167-4171

23. Kim HK, Kim JE, Chung J, Lee DS, Han KS, et al. Plasma level of stromal derived factor-1 (SDF-1) is increased in disseminated intravascular coagulation patients who have poor outcomes: in vitro effect of SDF-1 on coagulopathy. Thromb Res 2007;120(4): 559-566

24. Pérez-Morales RE, Del Pino MD, Valdivielso JM, Valdivielso JM, Ortiz A, et al. Inflammation in diabetic kidney disease. Nephron 2019;143(1): 1216

25. Ruster C, Wolf G. The role of chemokines and chemokine receptors in diabetic nephropathy. Front Biosci 2008;13: 944-955

26. Sayyed SG, Hägele H, Kulkarni OP, Endlich K, Segerer S, et al. Podocytes produce homeostatic chemokine stromal cell-derived factor-1/CXCL12, which contributes to glomerulosclerosis, podocyte loss and albuminuria in a mouse model of type 2 diabetes. Diabetologia 2009 Nov; $52(11)$ : 2445-2454

27. Alicic RZ, Rooney MT, Tuttle KR. Diabetic kidney disease: challenges, progress, and possibilities. Clin J Am Soc Nephrol 2017;12(12): 2032-2045

28. Scirica BM, Mosenzon O, Bhatt DL, Udell JA, Steg PG, et al. Cardiovascular outcomes according to urinary albumin and kidney disease in patients with type 2 diabetes at high cardiovascular risk: observations from the SAVOR-TIMI 53 Trial. JAMA Cardiol 2018;3(2): 155-163

29. Vijay S, Hamide A, Senthilkumar GP, Mehalingam V. Utility of urinary biomarkers as a diagnostic tool for early diabetic nephropathy in patients with type 2 diabetes mellitus. Diabetes Metab Syndr 2018;12(5): 649-652 
30. Newman DJ, Thakkar H, Edwards RG, Wilkie M, White T, et al. Serum cystatin C measured by automated immunoassay: a more sensitive marker of changes in GFR than serum creatinine. Kidney Int 1995;47(1): 312-318

31. Zhou B, Zou H, Xu G. Clinical utility of serum cystatin C in predicting diabetic nephropathy among patients with diabetes mellitus: a MetaAnalysis. Kidney Blood Press Res 2016;41(6): 919-928

32. Luo Q, Zhang B, Kuang D, Song G. Role of stromal-derived factor-1 in mesenchymal stem cell paracrine-mediated tissue repair. Curr Stem Cell Res Ther 2016;11: 585-592.

33. Panchapakesan U, Mather A, Pollock C. Role of GLP-1 and DPP-4 in diabetic nephropathy and cardiovascular disease. Clin Sci (Lond) 2013;124: $17-26$

34. Zhong J, Maiseyeu A, Davis SN, Rajagopalan S. DPP4 in cardiometabolic disease: recent insights from the laboratory and clinical trials of DPP4 inhibition. Circ Res 2015;116: 1491-1504.

35. Negro R, Greco EL, Greco G. Active stromal cell-derived factor 1a and endothelial progenitor cells are equally increased by alogliptin in good and poor diabetes control. Clin Med Insights Endocrinol Diabetes 2017;10: 1179551417743980

36. Li F, Chen J, Leng F, Lu Z, Ling Y. Effect of saxagliptin on circulating endothelial progenitor cells and endothelial function in newly diagnosed type 2 diabetic patients. Exp Clin Endocrinol Diabetes 2017;125(6): 400-407

37. Papazafiropoulou AK, Papanas N, Trikkalinou A, Fousteris E, Melidonis A. The oral dipeptidyl-peptidase-4 inhibitor sitagliptin increases circulating levels of stromal-derived factor-1 alpha. Exp Clin Endocrinol Diabetes 2018;126(6): 367-370

38. Broxmeyer HE, Capitano M, Campbell TB, Hangoc G, Cooper S. Modulation of hematopoietic chemokine effects in vitro and in vivo by DPP4/CD26. Stem Cells Dev 2016;25(8): 575-585

39. Packer M. Have dipeptidyl peptidase- 4 inhibitors ameliorated the vascular complications of type 2 diabetes in large-scale trials? The potential confounding effect of stem-cell chemokines. Cardiovasc Diabetol 2018;17(1): 9

\section{Tables}

Table 1 Clinical characteristics of the study participants 


\begin{tabular}{|c|c|c|c|c|c|c|c|}
\hline Variables & Controls & $\mathrm{T} 2 \mathrm{D}$ & & & & & $\begin{array}{l}P \text { for } \\
\text { trend }\end{array}$ \\
\hline & & Total & Q1 & Q2 & Q3 & Q4 & \\
\hline SDF-1 & $2.98 \pm 1.36$ & $4.17 \pm 1.87^{\star \star \star}$ & $<2.95$ & $2.95-3.92$ & $3.94-5.40$ & $>5.40$ & \\
\hline$n$ & 42 & 171 & 43 & 44 & 42 & 42 & \\
\hline Age (years) & $54.38 \pm 13.14$ & $55.22 \pm 12.37$ & $50.49 \pm 14.10$ & $51.98 \pm 11.55$ & $59.55 \pm 9.73$ & $59.10 \pm 11.25$ & $0.000 \# \# \#$ \\
\hline Male, n (\%) & $21(50)$ & $113(66.1)$ & $28(65.1)$ & $33(75.0)$ & 25(59.5) & $27(64.3)$ & 0.485 \\
\hline Diabetic duration (years) & $\mathrm{N}$ & $5.0(0-10.0)$ & $4.0(0-10.0)$ & $4(0.25-10.0)$ & $5.5(0-10)$ & $7.5(1-10)$ & 0.464 \\
\hline BMI $\left(\mathrm{kg} / \mathrm{m}^{2}\right)$ & $24.87 \pm 4.40$ & $25.49 \pm 4.12$ & $25.22 \pm 3.65$ & $25.64 \pm 3.91$ & $24.76 \pm 4.27$ & $26.29 \pm 4.61$ & 0.391 \\
\hline SBP $(\mathrm{mmHg})$ & $\begin{array}{l}125(130.5- \\
142.75)\end{array}$ & 133(124-133) & $132(121-146)$ & $\begin{array}{l}133(125- \\
144.50)\end{array}$ & $\begin{array}{l}\text { 134(127.5- } \\
150.25)\end{array}$ & $\begin{array}{l}137(121.5- \\
150.5)\end{array}$ & 0.617 \\
\hline $\mathrm{DBP}(\mathrm{mmHg})$ & $80.03 \pm 10.40$ & $81.15 \pm 11.22$ & $82.86 \pm 9.63$ & $81.20 \pm 9.62$ & $79.60 \pm 11.74$ & $80.90 \pm 13.65$ & 0.613 \\
\hline \multicolumn{8}{|l|}{ Antidiabetic treatment } \\
\hline Insulin treatment, n (\%) & $\mathrm{N}$ & $39(22.8)$ & $8(18.6)$ & $11(25.0)$ & $11(26.2)$ & $9(21.4)$ & 0.833 \\
\hline Metformin, n (\%) & $\mathrm{N}$ & $63(36.8)$ & 19(44.2) & $15(34.1)$ & $10(23.8)$ & 19(45.2) & 0.140 \\
\hline Acarbose, n (\%) & $\mathrm{N}$ & $14(8.2)$ & $5(11.6)$ & $1(2.3)$ & $4(9.5)$ & $4(9.5)$ & 0.403 \\
\hline $\begin{array}{l}\text { Insulin-secretagogues, } \mathrm{n} \\
(\%)\end{array}$ & $\mathrm{N}$ & $53(31.0)$ & 13(30.2) & $13(29.5)$ & $11(26.2)$ & $16(38.2)$ & 0.683 \\
\hline Insulin-sensitisers, n (\%) & $\mathrm{N}$ & $15(8.8)$ & $2(4.7)$ & $7(15.9)$ & $3(7.1)$ & $3(7.1)$ & 0.262 \\
\hline DPP-4 inhibitors, n (\%) & $\mathrm{N}$ & $4(2.3)$ & $3(7.0)$ & $1(2.3)$ & $0(0)$ & $0(0)$ & 0.109 \\
\hline Lg (Urinary albumin) & $\mathrm{N}$ & $1.34 \pm 0.76$ & $1.27 \pm 0.74$ & $1.22 \pm 0.65$ & $1.21 \pm 0.67$ & $1.65 \pm 0.88$ & $0.031 \#$ \\
\hline Lg (UACR) & $\mathrm{N}$ & $1.47 \pm 0.75$ & $1.38 \pm 0.68$ & $1.37 \pm 0.63$ & $1.34 \pm 0.68$ & $1.79 \pm 0.92$ & $0.021 \#$ \\
\hline \multicolumn{8}{|l|}{ Categories of proteinuria } \\
\hline $\begin{array}{l}\text { Normoalbuminuria, } \mathrm{n} \\
(\%)\end{array}$ & $\mathrm{N}$ & $106(62.0)$ & $31(72.1)$ & $31(70.5)$ & $27(64.3)$ & $17(40.5)$ & $0.029 \#$ \\
\hline Microalbuminuria, n (\%) & $\mathrm{N}$ & $45(26.3)$ & $9(20.9)$ & $9(20.5)$ & $12(28.6)$ & 15(35.7) & $0.029 \#$ \\
\hline Macroalbuminuria, n (\%) & $\mathrm{N}$ & $20(11.7)$ & $3(7.0)$ & $4(9.1)$ & $3(7.1)$ & $10(23.8)$ & $0.029 \#$ \\
\hline HbA1c (\%) & $5.92 \pm 0.41$ & $9.38 \pm 2.20 * \star \star$ & $9.06 \pm 2.12$ & $8.91 \pm 2.18$ & $9.94 \pm 2.22$ & $9.64 \pm 2.18$ & 0.100 \\
\hline $\operatorname{ALT}(\mathrm{U} / \mathrm{L})$ & $16(12-26)$ & $21(14-31)$ & $17(13-31.5)$ & $\begin{array}{l}24(16.75- \\
32.25)\end{array}$ & 18(10.5-29.75) & $21.5(14-33.75)$ & 0.387 \\
\hline AST (U/L) & $18(15-20)$ & $18(14-24)$ & $15.5(13-22)$ & $18(15.5-22.5)$ & 18(13.5-24) & $19(14-29)$ & 0.167 \\
\hline $\mathrm{ADA}(\mathrm{U} / \mathrm{L})$ & $9(7-10)$ & $10(8-14)^{\star \star}$ & $10(8-12)$ & $9(8-12)$ & $11(9-15.25)$ & $12(10-16)$ & $0.004 \# \#$ \\
\hline TG (mmol/L) & $\begin{array}{l}1.02(0.85- \\
1.66)\end{array}$ & $\begin{array}{l}1.66(1.14- \\
3.03)^{\star \star}\end{array}$ & $\begin{array}{l}1.68(1.19- \\
3.08)\end{array}$ & $\begin{array}{l}1.81(1.09- \\
2.79)\end{array}$ & $\begin{array}{l}1.43(0.98- \\
2.48)\end{array}$ & $\begin{array}{l}1.94(1.21- \\
4.08)\end{array}$ & 0.504 \\
\hline $\mathrm{TC}(\mathrm{mmol} / \mathrm{L})$ & $4.49 \pm 1.23$ & $4.69 \pm 1.31$ & $4.63 \pm 1.10$ & $4.56 \pm 0.92$ & $4.58 \pm 0.95$ & $4.96 \pm 1.95$ & 0.477 \\
\hline HDL-c (mmol/L) & $1.23 \pm 0.33$ & $1.19 \pm 0.24$ & $1.15 \pm 0.24$ & $1.18 \pm 0.24$ & $1.24 \pm 0.27$ & $1.19 \pm 0.21$ & 0.430 \\
\hline LDL-c (mmol/L) & $2.72 \pm 0.95$ & $2.88 \pm 0.86$ & $2.91 \pm 0.85$ & $2.85 \pm 0.85$ & $2.83 \pm 0.83$ & $2.94 \pm 0.92$ & 0.935 \\
\hline BUN (mmol/L) & $5.08 \pm 1.38$ & $5.45 \pm 1.82$ & $4.88 \pm 1.44$ & $5.24 \pm 1.44$ & $5.23 \pm 1.55$ & $6.42 \pm 2.32$ & $0.001 \# \#$ \\
\hline $\mathrm{Cr}$ (umol/L) & $58(47.5-66.5)$ & $56(49.2-67)$ & $\begin{array}{l}54.5(44.25- \\
64.75)\end{array}$ & $\begin{array}{l}57(50.5- \\
66.75)\end{array}$ & $54(48.5-65.5)$ & $59(53.5-79.5)$ & 0.077 \\
\hline Serum UA (umol/L) & $293.41 \pm 88.03$ & $326.6 \pm 102.33$ & $318.38 \pm 109.59$ & $314.73 \pm 95.14$ & $323.23 \pm 103.17$ & $350.77 \pm 101.05$ & 0.390 \\
\hline Cystatin C (mg/L) & $\begin{array}{l}0.71(0.58- \\
0.82)\end{array}$ & $\begin{array}{l}0.71(0.58- \\
0.86)\end{array}$ & $\begin{array}{l}0.68(0.53- \\
0.80)\end{array}$ & $\begin{array}{l}0.64(0.54- \\
0.72)\end{array}$ & $\begin{array}{l}0.81(0.57- \\
0.98)\end{array}$ & $\begin{array}{l}0.79(0.69- \\
1.14)\end{array}$ & $0.000 \# \# \#$ \\
\hline eGFR $\left(\mathrm{ml} / \mathrm{min} / 1.73 \mathrm{~m}^{2}\right)$ & $115.31 \pm 25.47$ & $110.98 \pm 28.83$ & $123.84 \pm 24.74$ & $117.83 \pm 21.65$ & $106.91 \pm 28.81$ & $94.98 \pm 31.47$ & $0.000 \# \# \#$ \\
\hline
\end{tabular}




\begin{tabular}{|c|c|c|c|c|c|c|c|}
\hline DKD, n (\%) & N & $24(14.0)$ & $3(7.0)$ & $5(11.4)$ & $5(11.9)$ & $11(26.2)$ & 0.062 \\
\hline $\mathrm{ESR}(\mathrm{mm})$ & $\begin{array}{l}5.5(2.25- \\
13.25)\end{array}$ & $6(3-10)$ & $5.5(2-9.5)$ & $5(2-9)$ & 7(3-14) & $7(5-17)$ & $0.029 \#$ \\
\hline CRP (mg/L) & $\begin{array}{l}0.12(0.01- \\
1.77)\end{array}$ & $\begin{array}{l}0.71(0.58- \\
0.86)\end{array}$ & $\begin{array}{l}0.17(0.05- \\
0.80)\end{array}$ & $\begin{array}{l}0.24(0.05- \\
1.39)\end{array}$ & $\begin{array}{l}0.56(0.08- \\
3.18)\end{array}$ & $\begin{array}{l}0.34(0.11- \\
6.14)\end{array}$ & 0.382 \\
\hline APTT (s) & $29.26 \pm 4.87$ & $28.34 \pm 2.62$ & $29.12 \pm 3.64$ & $28.97 \pm 2.30$ & $27.74 \pm 2.07$ & $27.69 \pm 2.00$ & $0.043 \#$ \\
\hline $\mathrm{Fg}(\mathrm{g} / \mathrm{L})$ & $\begin{array}{l}2.56(2.20- \\
3.19)\end{array}$ & $\begin{array}{l}2.36(2.18- \\
2.94)\end{array}$ & $\begin{array}{l}2.34(2.08- \\
2.82)\end{array}$ & $\begin{array}{l}2.31(2.01- \\
2.80)\end{array}$ & $\begin{array}{l}2.35(2.23- \\
2.88)\end{array}$ & $\begin{array}{l}2.61(2.19- \\
3.31)\end{array}$ & 0.198 \\
\hline D-dimer (ug/L) & $243(190-330)$ & $220(190-380)$ & $\begin{array}{l}190(190- \\
462.5)\end{array}$ & $190(160-265)$ & $220(190-360)$ & $325(195-610)$ & 0.008\#\# \\
\hline $\operatorname{PLT}\left({ }^{*} 10^{9} / \mathrm{L}\right)$ & $\begin{array}{l}206.5(175.5- \\
259.5)\end{array}$ & $\begin{array}{l}203.5(169- \\
238.5)\end{array}$ & $\begin{array}{l}207(170.25- \\
207)\end{array}$ & $\begin{array}{l}213.5(189.5- \\
239.75)\end{array}$ & $\begin{array}{l}\text { 189(159- } \\
233.5)\end{array}$ & $\begin{array}{l}208.5(163.5- \\
250.75)\end{array}$ & 0.421 \\
\hline WBC $\left({ }^{*} 10^{9} / \mathrm{L}\right)$ & $5.84 \pm 1.66$ & $6.62 \pm 1.90 *$ & $6.47 \pm 1.84$ & $6.64 \pm 1.38$ & $6.22 \pm 1.37$ & $7.14 \pm 2.65$ & 0.166 \\
\hline NEU (\%) & $56.48 \pm 8 / 29$ & $60.58 \pm 9.51^{*}$ & $59.19 \pm 9.34$ & $60.66 \pm 9.60$ & $58.70 \pm 8.77$ & $63.79 \pm 9.78$ & 0.071 \\
\hline
\end{tabular}

Normally distributed values in the table are given as the mean \pm SD, skewed distributed values are given as the median ( 25 and $75 \%$ interquartiles), and categorical variables are given as frequency (percentage)

SDF-1 stromal cell-derived factor-1, BMI body mass index, SBP/DBP systolic/diastolic blood pressure, UACR urine albumin/creatinine ratio, $\mathrm{HbA}_{1 \mathrm{c}}$ glycosylated hemoglobin A1c, ALT alanine transaminase, AST aspartate aminotransferase, ADA adenosine deaminase, TG triglyceride, TC total cholesterol, HDL-c high density lipoprotein cholesterol, LDL-c low density lipoprotein cholesterol, BUN blood urea nitrogen, Cr creatinine, Serum UA serum uric acid, eGFR estimated glomerular filtration rate, DKD diabetic kidney disease, ESR erythrocyte sedimentation rate, CRP C-reactive protein, APTT activated partial thromboplastin time, Fg fibrinogen, PLT platelet, WBC white blood cell count, NEU neutrophil percentage

ANOVA, the Kruskal-Wallis test and the Chi squared test were conducted to determine $p$ values for normally distributed continuous variables, skewed continuous variables and categorical variables, respectively. \# $P<0.05, \# \# P<0.01, \# \# \#<0.001$

$\star P<0.05, * \star P<0.01, * \star \star * P<0.001$, the comparison of T2D with Controls

Table 2 Relationship between SDF-1 and clinical parameters in patients with T2D 


\begin{tabular}{|lll|}
\hline Variables & $r$ & $P$ value \\
\hline Lg (Urinary albumin) & 0.262 & 0.001 \\
\hline Lg (UACR) & 0.276 & 0.000 \\
\hline Age & 0.256 & 0.001 \\
\hline HbA1c & 0.179 & 0.019 \\
\hline ADA & 0.262 & 0.001 \\
\hline BUN & 0.353 & 0.000 \\
\hline Cr & 0.154 & 0.053 \\
\hline Cystatin C & 0.330 & 0.000 \\
\hline eGFR & -0.368 & 0.000 \\
\hline DKD & 0.186 & 0.015 \\
\hline ESR & 0.262 & 0.003 \\
\hline CRP & 0.151 & 0.131 \\
\hline APTT & -0.294 & 0.001 \\
\hline Fg & 0.114 & 0.207 \\
\hline D-dimer & 0.217 & 0.015 \\
\hline WBC & 0.145 & 0.067 \\
\hline NEU & 0.196 & 0.013 \\
\hline
\end{tabular}

rpearson's or spearman's correlation coefficient

Table 3 Multiple linear regression models displaying adjusted estimates for SDF-1 for outcomes of albuminuria indexes and eGFR adjusted for the other clinical covariates in each model in patients with T2D

\begin{tabular}{|lllll|}
\hline Models & $B(95 \% \mathrm{Cl})$ & $t$ & $p$ & $R^{2}$ for model \\
\hline Lg (Urinary albumin) & & & & \\
\hline Model 0 & $0.106(0.044$ to 0.169$)$ & 3.364 & 0.001 & 0.069 \\
\hline Model 1 & $0.103(0.037$ to 0.169$)$ & 3.099 & 0.002 & 0.100 \\
\hline Model 2 & $0.077(0.010$ to 0.143$)$ & 2.283 & 0.024 & 0.206 \\
\hline Model 3 & $0.076(0.007$ to 0.145$)$ & 2.185 & 0.031 & 0.207 \\
\hline Lg (UACR) & $0.112(0.050$ to 0.169$)$ & 3.582 & 0.000 & 0.076 \\
\hline Model 0 & $0.092(0.028$ to 0.156$)$ & 2.851 & 0.005 & 0.136 \\
\hline Model 1 & $0.068(0.003$ to 0.133$)$ & 2.057 & 0.042 & 0.242 \\
\hline Model 2 & $0.071(0.003$ to 0.130$)$ & 2.077 & 0.040 & 0.246 \\
\hline Model 3 & $-3.975(-6.306$ to -1.643$)$ & -3.375 & 0.001 & 0.378 \\
\hline eGFR & $-5.732(-8.164$ to -3.301$)$ & -4.661 & 0.000 & 0.129 \\
\hline Model 0 & $-3.489(-5.724$ to -1.255$)$ & -3.089 & 0.002 & 0.356 \\
\hline Model 1 & $-3.549(-5.848$ to -1.249$)$ & -3.055 & 0.003 & 0.368 \\
\hline Model 2 & -3.978 & & & \\
\hline Model 3 & & & & \\
\hline
\end{tabular}

Model 0: unadjusted model

Model 1: adjusted for age, male, diabetic duration, BMI 


\section{Figures}

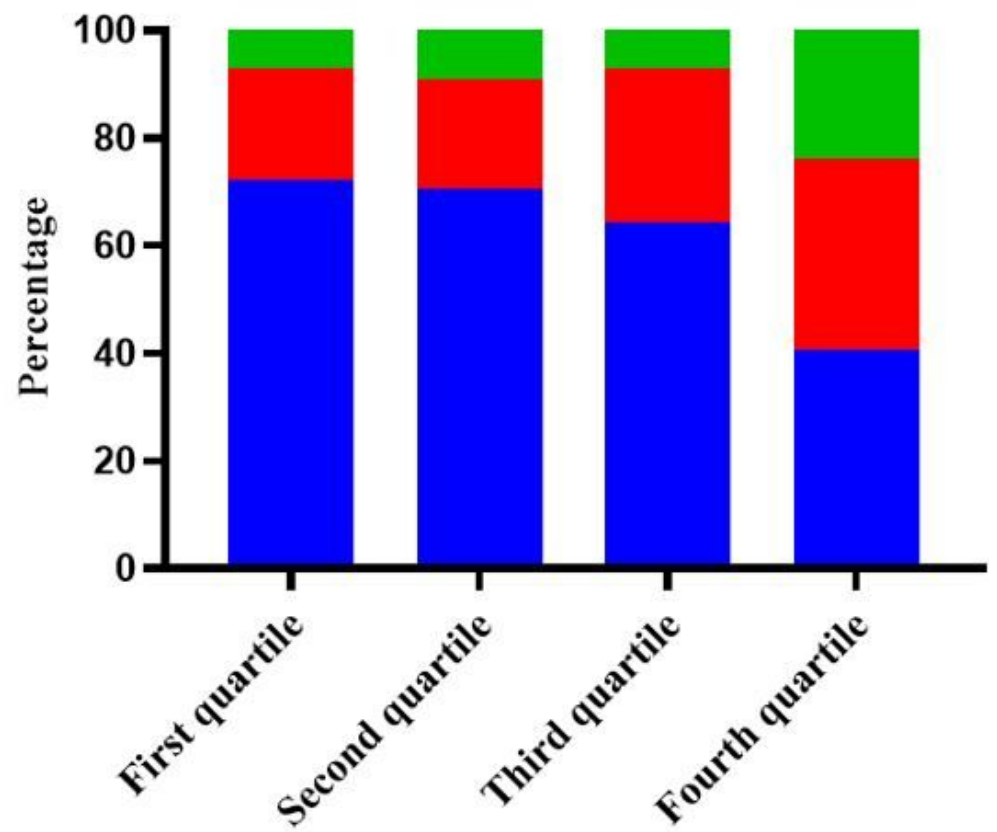

Macroalbuminuria

- Microalbuminuria

- Normoalbuminuria

Figure 1

The proportion of albuminuria types stratifed by SDF-1 quartiles 


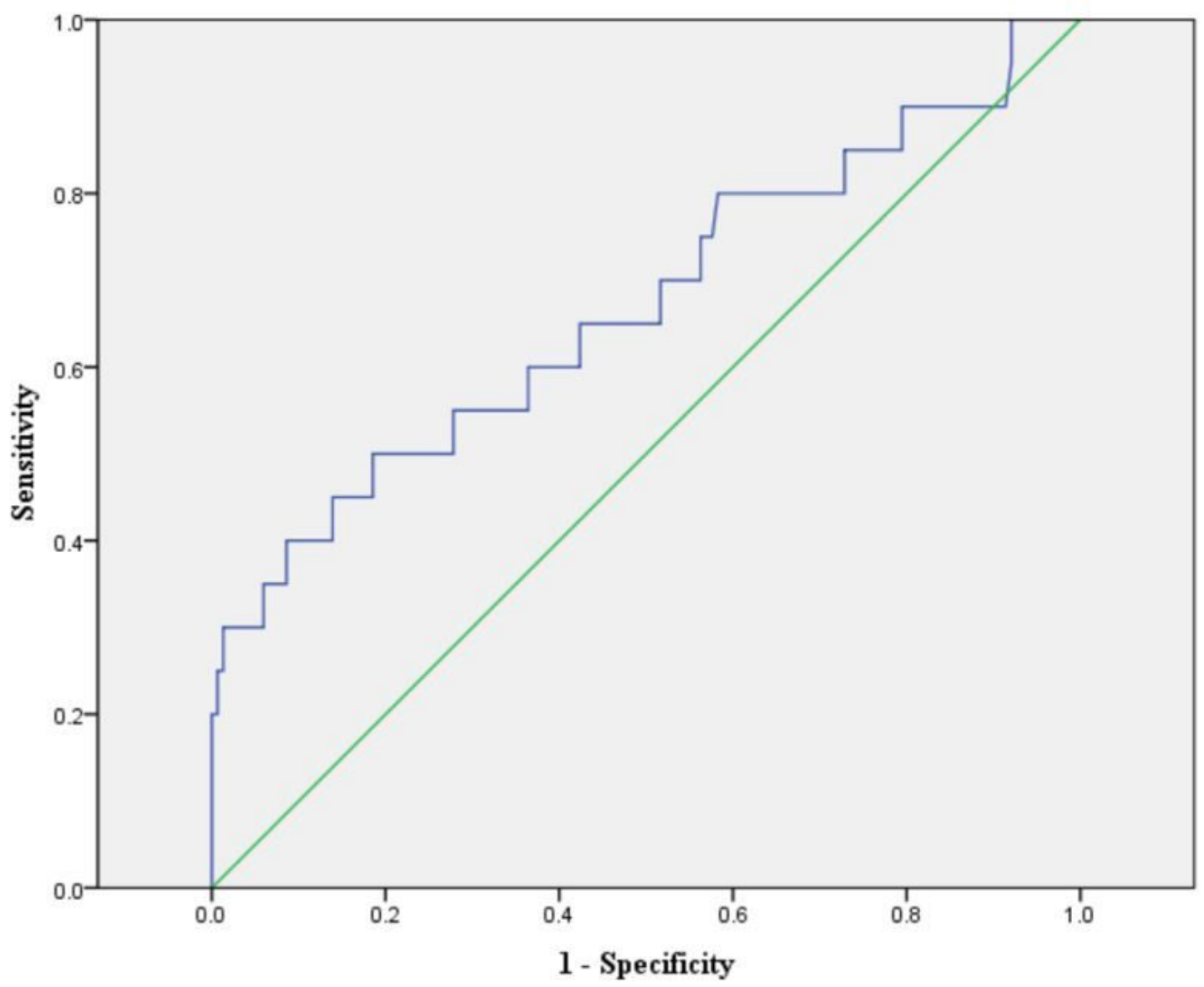

Figure 2

ROC analysis to analyze the ability of SDF-1 to indicate macroalbuminuria AUC of SDF-1 was 0.671 (95\% Cl 0.626-0.816). Optimal cutof value of SDF-1 was 5.735 to indicate macroalbuminuria; Youden index $=0.315$, sensitivity $=50.00 \%$ and specifcity $=81.46 \%$ 


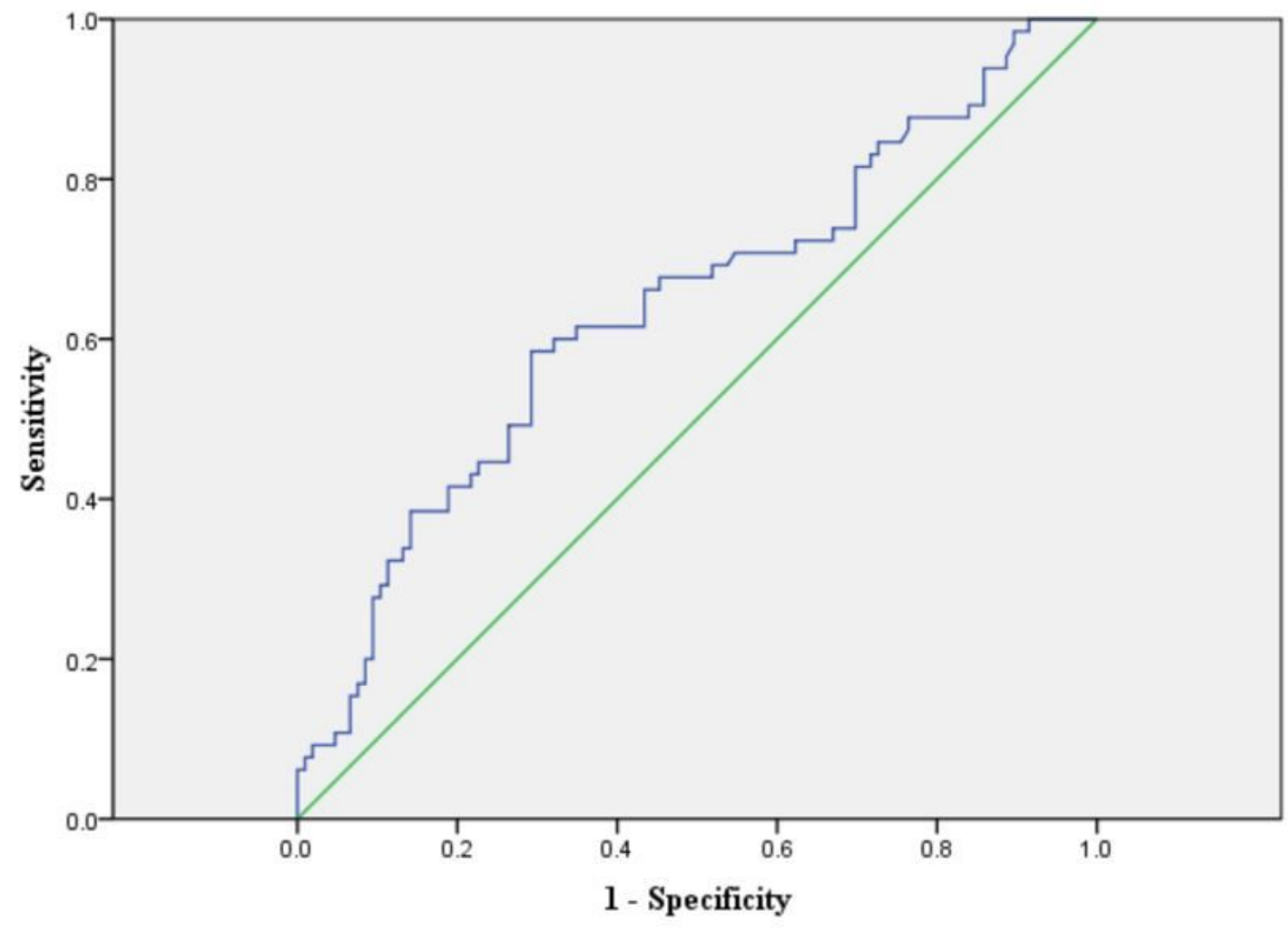

Figure 3

ROC analysis to analyze the ability of SDF-1 to indicate abnormal albuminuria AUC of SDF- 1 was 0.639 (95\% Cl $0.551-0.726)$. Optimal cutof value of SDF-1 was 4.321 to indicate abnormal albuminuria; Youden index $=0.292$, sensitivity $=58.46 \%$ and specifcity $=70.78 \%$ 


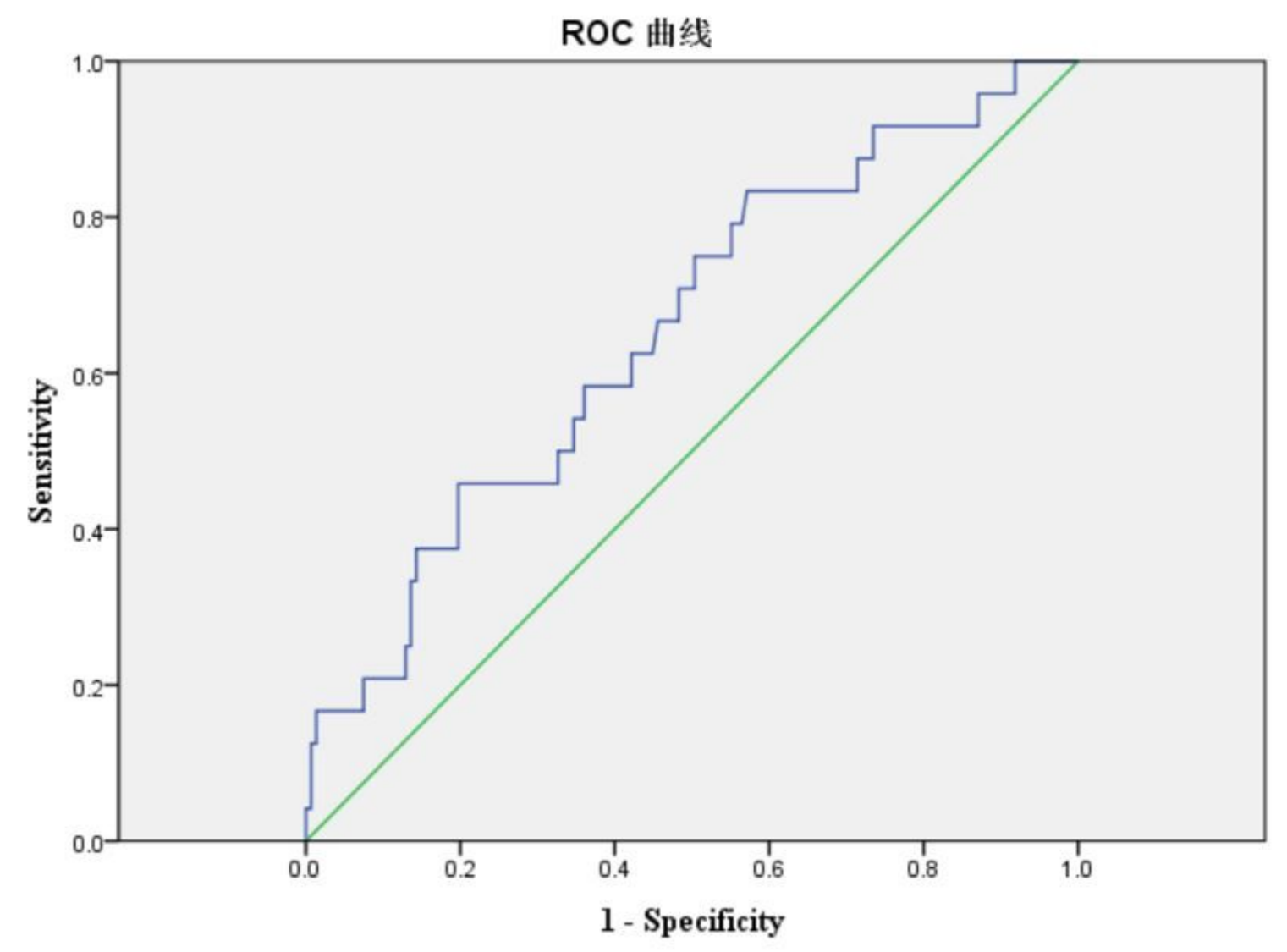

\section{Figure 4}

ROC analysis to analyze the ability of SDF-1 to indicate DKD AUC of SDF-1 was 0.654 ( $95 \%$ CI $0.536-0.773$ ). Optimal cutof value of SDF-1 was 3.505 to indicate DKD; Youden index $=0.262$, sensitivity $=83.33 \%$ and specifcity $=42.86 \%$ 